\title{
ChemComm
}

\section{Electrostatics and flexibility drive membrane recognition and early penetration by the antimicrobial peptide dendrimer bH1 $\dagger$}

\author{
Cite this: Chem. Commun., 2013, \\ 49, 8821 \\ Received 1st July 2013, \\ Accepted 1st August 2013
}

DOI: $10.1039 / c 3 c c 44912 b$

\author{
Harish K. Ravi, ${ }^{a}$ Michaela Stach, ${ }^{a}$ Thereza A. Soares, ${ }^{b}$ Tamis Darbre, ${ }^{a}$ \\ Jean-Louis Reymond ${ }^{a}$ and Michele Cascella*a
}

www.rsc.org/chemcomm

Molecular dynamics simulations of the polycationic antimicrobial peptide dendrimer bH1 (Leu $)_{8}(\text { DapLeu })_{4}(\text { DapPhe })_{2}$ DapLys- $\mathrm{NH}_{2}$ binding to membranes suggest that electrostatic interactions with the polyanionic lipopolysaccharide (LPS) and conformational flexibility of the 2,3-diaminopropanoic acid (Dap) branching units drive its selective insertion into microbial membranes.

Antibiotic resistant bacteria represent one of the major health care problems today, in particular Gram-negative pathogens such as Pseudomonas aeruginosa (PA), which are lethal to cystic fibrosis and immunocompromised patients. ${ }^{1}$ Recently, we reported the peptide dendrimer bH1 (Fig. 1) ${ }^{2}$ as a new type of membrane disrupting antimicrobial peptide $(\mathrm{AMP})^{3}$ potentially suitable to address antibiotic resistance (Fig. 1). Dendrimer bH1 shows potent antimicrobial activity against Gram-negative $\operatorname{E} . \operatorname{coli}(\mathrm{MIC}=1 \mu \mathrm{M})$ and $P$. aeruginosa $(\mathrm{MIC}=5 \mu \mathrm{M})$ without showing significant hemolysis $(\mathrm{MHC}>500 \mu \mathrm{M})$.

Most AMPs are polycationic amphiphilic sequences, which disrupt bacterial membranes by electrostatic binding to anionic groups of LPS or phospholipids followed by hydrophobicity driven membrane penetration, ${ }^{3}$ which may lead to membrane lysis or cell penetration and subsequent interaction with an intracellular target. ${ }^{4}$ To understand the selective interaction of bH1 with the LPS coated outer membrane of PA versus the eukaryotic cell membrane, we performed all atom molecular dynamics (MD) simulations of the dendrimer ${ }^{5-8}$ interacting with membrane models constituted of a 1-palmitoyl-2-oleoyl-sn-glycero-3-phosphocholine (POPC) bilayer as a model of the eukaryotic membrane, or of an asymmetric LPS on top of a 1,2-dipalmytoyl-3-phosphatydyl-ethanolamine (DPPE) layer as a model of the PA outer membrane (structure in ESI $\dagger$ ).

\footnotetext{
${ }^{a}$ Department of Chemistry and Biochemistry, University of Bern, Freiestrasse 3, 3012 Bern, Switzerland. E-mail: michele.cascella@iac.unibe.ch;

Fax: +4131631 3994; Tel: +41316314256

${ }^{b}$ Department of Fundamental Chemistry, Federal University of Pernambuco, Av. Jornalista Anibal Fernandes, $s / n^{\circ}$ Cidade Universitária 50740-560, Recife, Brazil $\dagger$ Electronic supplementary information (ESI) available: Chemical architecture of LPS, RESP charges for the branching Dap residue, interactions between bH1 and POPC membrane, computational and experimental protocols. See DOI: 10.1039/ c3cc44912b
}

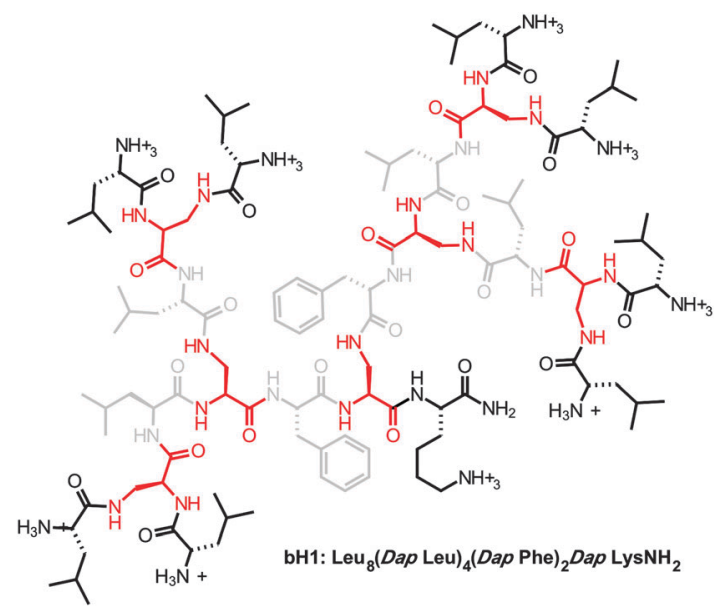

Fig. 1 Structural formula of antimicrobial peptide dendrimer bH1.

Standard MD protocols were followed using force field parameters and thermally equilibrated coordinates reported for POPC $^{9 a}$ and LPS. ${ }^{9 b}$

In both membrane systems bH1 approached the membrane surface in the first $10 \mathrm{~ns}$ of the simulation and established contacts via its multiple N-terminal ammonium groups. In bH1:POPC, the contacts involved one common terminal dendron Leu $_{2}$ Dap of bH1 binding to the phosphate groups of POPC present at the watermembrane interface. The dendrimer-membrane interface did not evolve significantly within the simulated time (500 ns) and the whole dendrimer structure remained fully solvated in the aqueous phase (ESI, $\uparrow$ Fig. S4).

In PA the outer phospho-saccharide chains of LPS assemble in a structured multilayer characterized by a high density of negative electrostatic charges, and a strong hydrophilic character. In our MD simulations, bH1 made contacts with the membrane surface within the first $15 \mathrm{~ns}$ and involved the positively charged $\mathrm{Leu}_{2}$ Dap terminal dendron and the solvent-exposed LPS 0 - $\beta$-D-glucose (0GB) and 0- $\alpha$-D-glucose (0GA) moieties (Fig. 2E). Further penetration occurred around $18 \mathrm{~ns}$ by transient formation of hydrogen bonds between 6 - $\alpha$-D-glucose (6GA) in the LPS and the terminal leucines attached to the same Dap unit following a similar 

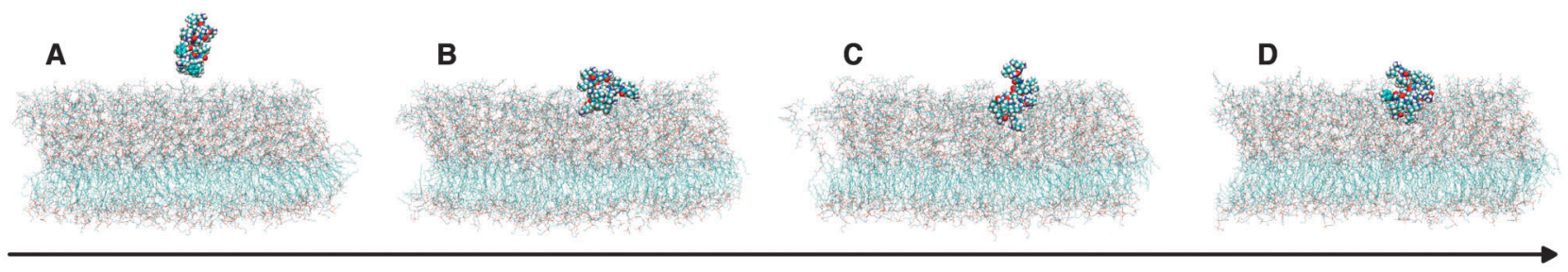

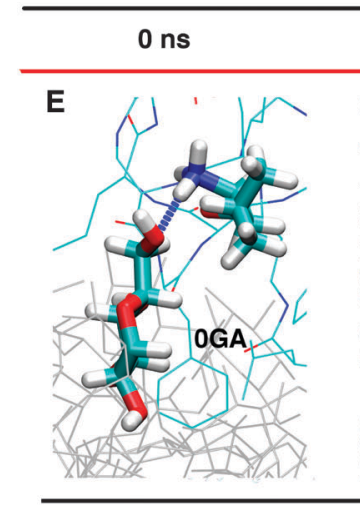

$15 \mathrm{~ns}$

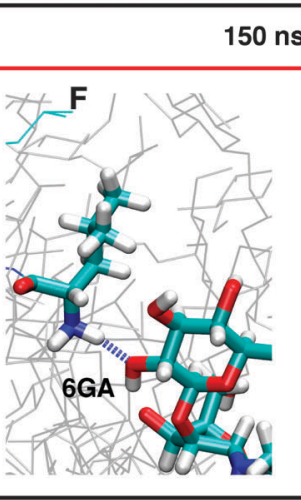

$25 \mathrm{~ns}$

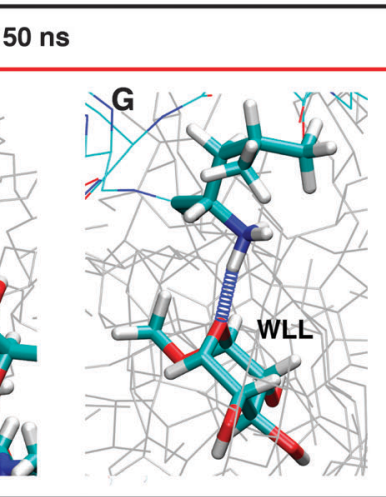

$60 \mathrm{~ns}$
200 ns

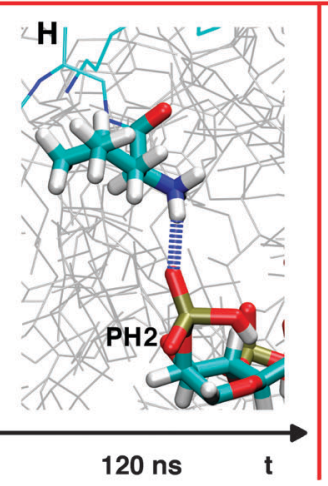

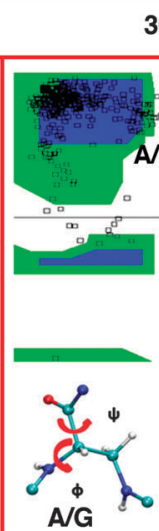

$300 \mathrm{~ns}$

t

I

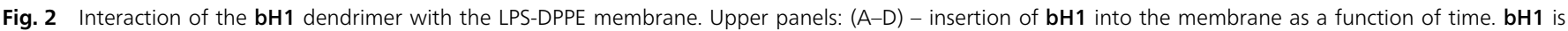

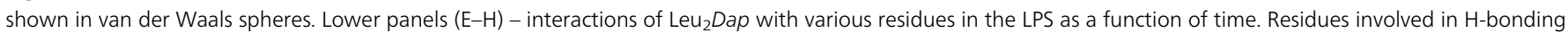

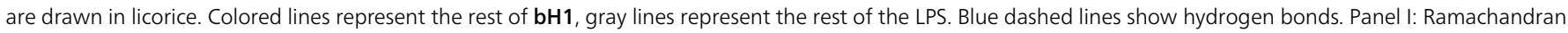

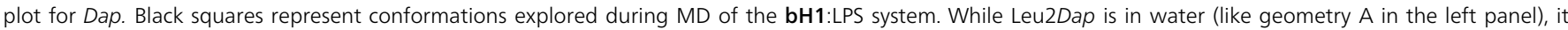

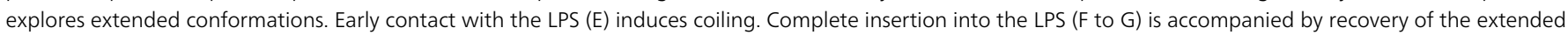
conformation, kept along the rest of the simulation ( $H, B, C$ and $D)$.

hydrogen bond acceptor-donor pattern as before (Fig. 2F). Between 20 and 120 ns bH1 underwent a major conformational change facilitating the insertion of a second $\mathrm{Leu}_{2}$ Dap dendron into the LPS, concomitant with deeper penetration of the leucines of the first $\mathrm{Leu}_{2}$ Dap dendron to $\sim 18 \AA$ from the water-LPS interface, enabling its anchoring to the L-glycero-D-manno-heptose-7-formamide (3H1) and phosphorylated 2-(2-hydroxyethyl)-6-deoxy-D-manno-heptose (PH2) of the LPS, whereby the three anionic phosphate groups of $\mathrm{PH} 2$ formed hydrogen bonds with the N-terminal ammonium groups of bH1 (Fig. 2H).

The contacts of the $\mathrm{Leu}_{2}$ Dap dendrons to $3 \mathrm{H} 1$ and $\mathrm{PH} 2$ were very stable, and no further penetration occurred within our simulation time (300 ns). Rather, the deep rooting of the first $\mathrm{Leu}_{2} \mathrm{Dap}$ dendron into the LPS induced penetration of the other ones. In particular, after about $100 \mathrm{~ns}$, one of the other three remaining $\mathrm{Leu}_{2}$ Dap dendrons started to interact with the outer-core of the LPS, and then followed a similar hydrogen-bonding pattern observed for the initial anchoring $\mathrm{Leu}_{2}$ Dap dendron. At the end of the $\mathrm{MD}$ simulation this second $\mathrm{Leu}_{2}$ Dap dendron had penetrated well inside the outer core of the LPS and its terminal leucines were just $6 \AA$ away from the inner core of the LPS.

Monitoring the insertion of the third and the fourth $\mathrm{Leu}_{2}$ Dap dendrons, we observed a hydrogen-bonding pattern with the outer core identical to that of the first two dendrons. Translocation of the various terminal $\mathrm{Leu}_{2}$ Dap dendrons of $\mathbf{b H} \mathbf{1}$ from the outer to the inner region of the LPS occurred on average in $100 \mathrm{~ns}$ by a diffusive mechanism in which the dendrimer built relatively weak and labile contacts with the various sugar moieties, in contrast to the initial insertion of the $\mathrm{Leu}_{2}$ Dap dendrons into the LPS, which required some activation.
The Dap residue of the terminal $\mathrm{Leu}_{2}$ Dap dendrons underwent significant conformational changes in the course of the MD simulation. Initially the dendrimer was fully hydrated and the dendrons assumed an extended conformation in which the Dap residue was present in the allowed $\beta$-sheet region of the Ramachandran plot (Fig. 2). Upon localization at the membranewater interface, this extended conformation was destabilized and Dap assumed a coiled conformation typical for a right-handed $\alpha$-helix. The $\beta$-branch of the asymmetric $\operatorname{Leu}_{2}$ Dap dendron is longer by one methylene group and conformationally more flexible than the $\alpha$-branch, and was always the first to penetrate the LPS membrane, followed by Dap and the shorter $\alpha$-branch. After the $\mathrm{Leu}_{2}$ Dap dendron had passed the region defined by the 0GB and 0GA saccharides and insertion into the LPS was complete, the secondary structure of Dap changed back to an extended conformation similar to that in the aqueous phase. Penetration of bH1 into the LPS thus seems to be favored by the conformational flexibility of the branching units, which allow local coiling and optimization of the interaction with the LPS at the water-membrane interface.

Electrostatic attraction between the positively charged terminal ends and complementary negatively charged phosphate groups provided the driving force inducing penetration of bH1 into the LPS during MD. The dendrimer insertion occurred through an entropy-driven process, with sequential binding of the different branches. The first layer of phosphate groups present in the LPS at about $18 \AA$ from the membrane-water interface constituted the main basin of attraction for the terminal $\mathrm{Leu}_{2}$ Dap dendrons. The simulated time (300 ns) was sufficient to observe localization of the dendrimer in this area of the LPS. The presence of a second 

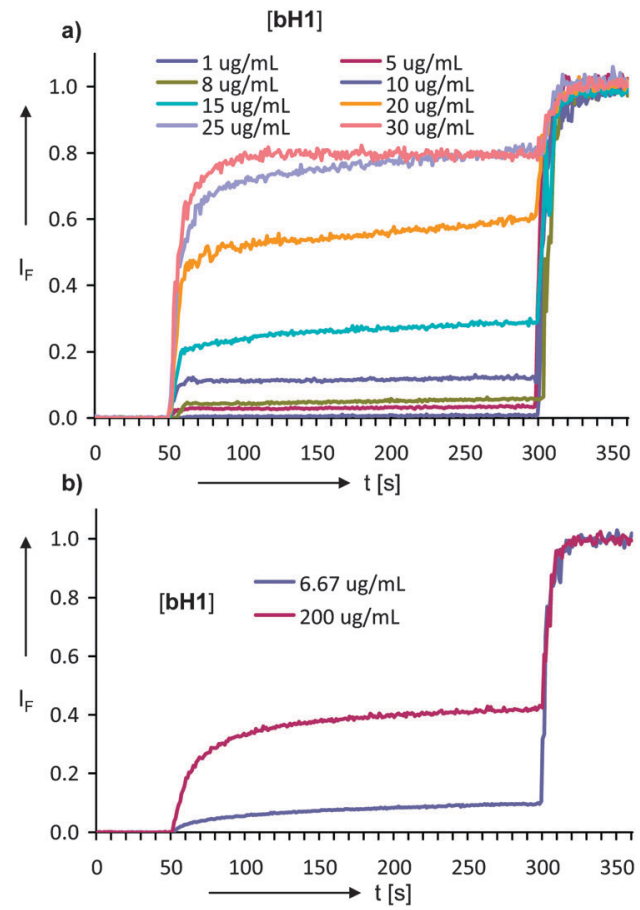

Fig. 3 bH1 induced CF-release from large unilamellar vesicles. (a) Phosphatidylglycerol (PG) LUV. (b) Phosphatidylcholine (PC) LUV.

layer of phosphates roughly $26 \AA$ deeper in the LPS may trigger further penetration in longer timescales.

The importance of electrostatic complementarity for the activity of bH1 was evidenced earlier by the observation that the uncharged, N-acetylated analog of bH1 shows no antibacterial or membrane disrupting effect. ${ }^{2}$ To further test this interaction, bH1 was exposed to 5(6)-carboxyflourescein (CF) loaded large unilamellar vesicles (LUVs) composed of phosphatidylglycerol (PG) as head groups, and induced CF release at low concentration $\left(1-30 \mu \mathrm{g} \mathrm{mL} \mathrm{m}^{-1}\right)$ demonstrating an interaction and disruption of this membrane. In contrast, LUVs composed of phosphatidylcholine (PC) head groups showed only partial release of CF upon exposure to bH1 up to $200 \mu \mathrm{g} \mathrm{mL} L^{-1}$, showing that bH1 does not interact with zwitterionic groups (Fig. 3). bH1 also shows high potency against Gram positive Bacillus subtilis $(\mathrm{MIC}=1 \mu \mathrm{M}){ }^{2}{ }^{2}$ Based on the present simulation we predict that bH1 can interact with negatively charged peptidoglycan elements, like teichoic acids, which are key to the structural stability of the outer cell wall.

In conclusion, the driving force facilitating penetration of the antimicrobial peptide dendrimer bH1 in the LPS of PA during MD was provided by electrostatic attractions exerted by the phosphate groups localized at the inner regions of the LPS layer on the external side of the membrane, which were established after initial membrane adsorption and sequential insertion of the four terminal $\mathrm{Leu}_{2}$ Dap dendrons, an event requiring local coiling of the branching Dap amino acid to facilitate adaptation to the geometrical restraints at the membrane-water interface. The observation of these coiling events at the interface-crossing point suggests that flexible branching units are crucial to the antimicrobial activity of peptide dendrimers, a finding which may be useful in future designs. Note that in the simulation time presented here the LPS bilayer was still well organized and only marginally perturbed by the dendrimer. It is likely that the antimicrobial function of bH1 is associated with phenomena occurring at longer timescales, possibly comprising aggregation of multiple bH1 units into the LPS. A crucial role played by aggregation has been already described for membrane-binding antibacterial linear peptides. ${ }^{10}$ Future studies addressing longer MD simulations, also with a higher concentration of bH1 molecules, may be able to elucidate this other aspect of the biological activity of peptide dendrimers.

This research was supported by the Swiss National Science Foundation (Grant No.: PP00P2_139195 for MC and 200020_ 140349 for JLR), and by the Marie-Curie ITN BioChemLig (Grant No.: FP7-ITN-238434). Partial computational resources were provided by the Environmental Molecular Sciences Laboratory at Pacific Northwest National Laboratory, WA, USA.

\section{Notes and references}

1 (a) R. M. Aris, P. H. Gilligan, I. P. Neuringer, K. K. Gott, J. Rea and J. R. Yakaskas, Am. J. Respir. Crit. Care Med., 1997, 155, 1699-1704; (b) A. Harris, C. Torres-Viera, L. Venkataraman, P. DeGirolami, M. Samore and M. Y. Carmeli, Clin. Infect. Dis., 1997, 28, 1128-1133; (c) P. Hsueh, P. L. Teng, P. Yang, U. Chen, S. Ho and K. Luh, J. Clin. Microbiol., 1998, 36, 1347-1351.

2 (a) M. Stach, N. Maillard, R. U. Kadam, D. Kalbermatter, M. Meury, M. G. P. Page, D. Fotiadis, T. Darbre and J.-L. Reymond, MedChemComm, 2012, 3, 86-89; (b) T. Darbre and J.-L. Reymond, Org. Biomol. Chem., 2012, 10, 1483-1492.

3 M. Bruschi, G. Pirri, A. Giuliani, S. F. Nicoletto, I. Baster, M. A. Scorciapino, M. Casu and A. C. Rinaldi, Peptides, 2010, 31, 1459-1467.

4 K. A. Brogden, Nat. Rev. Microbiol., 2005, 3, 328-350.

5 S. V. Lyulin, A. Darinskii and S. V. Lyulin, Phys. Rev. E, 2008, 78, 041801.

6 G. M. Pavan, A. Barducci, L. Albertazzi and M. Parrinello, Soft Matter, 2013, 9, 2593-2597.

7 P. Welch and M. Muthukumar, Macromolecules, 2000, 33, 6159-6167.

8 K. Karatasos, D. B. Adolf and G. R. Davies, J. Chem. Phys., 2001, 115, $5310-5318$.

9 (a) A. V. Vargiu, F. Collu, R. Schulz, K. M. Pos, M. Zacharias, U. Kleinekathöfer and P. Ruggerone, J. Am. Chem. Soc., 2011, 28, 10704-10708; (b) K. N. Kirschner, R. D. Lins, A. Maass and T. A. Soares, J. Chem. Theory Comput., 2012, 8, 4719-4731.

10 (a) R. E. Hancock and D. S. Chapple, Antimicrob. Agents Chemother., 1999, 43, 1317-1323; (b) K. Matsuzaki, Biochim. Biophys. Acta, 1998, 1376, 391-400; (c) Z. Oren and Y. Shai, Biopolymers, 1998, 47, 451-463; (d) Y. Bai, S. P Liu, J. G. Li, R. Lakshminarayanan, P. Sarawathi, C. Tang, D. C. Ho, C. Verma, R. W. Bauerman and K. Perushin, J. Biol. Chem., 2012, 287, 26606-26617; (e) M. M. Domingues, N. C. Santos and M. A. R. B. Castanho, Curr. Protein Pept. Sci., 2012, 7, 611-619; (f) S. Bobone, Y. Gerelli, M. De Zotti, G. Bocchinfuso, A. Farrotti, B. Orioni, F. Sebastiani, E. Latter, J. Penfold, R. Senesi, F. Formaggio, A. Palleschi, C. Toniolo, G. Fragneto and L. Stella, Biochim. Biophys. Acta, 2013, 1828, 1013-1024; (g) V. P. Zhodanov and F. Hook, Phys. Rev. E, 2013, 87, 04718. 